\title{
A Comparative Evaluation of Commercially Available Cell- Based Allografts in a Rat Spinal Fusion Model
}

\author{
BRIAN JOHNSTONE, PHD,${ }^{1}$ NIANLI ZHANG, PHD,${ }^{2}$ ERIK I. WALDORFF, PHD, ${ }^{2}$ ERIC SEMLER, PHD, ${ }^{3}$ \\ ANOUSKA DASGUPTA, PHD,${ }^{3}$ MARCEL BETSCH, MD,${ }^{1}$ PAOLO PUNSALAN, MD,${ }^{1}$ HOLLY CHO, MS, ${ }^{1}$ \\ JAMES T. RYABY, PHD ${ }^{2}$ JUNG YOO, MD ${ }^{1}$ \\ ${ }^{I}$ Department of Orthopaedics and Rehabilitation, Oregon Health \& Science University, Portland, Oregon, ${ }^{2}$ Orthofix Medical Inc, Lewisville, Texas, ${ }^{3}$ MTF \\ Biologics, Edison, New Jersey
}

\begin{abstract}
Background: To evaluate the comparative abilities of commercially available, viable, cellular bone allografts to promote posterolateral spinal fusion.

Methods: Human allografts containing live cells were implanted in the athymic rat model of posterolateral spine fusion. Three commercially available allogeneic cellular bone matrices (Trinity Evolution, Trinity ELITE and Osteocel Plus) were compared with syngeneic iliac crest bone as the control. All spines underwent radiographs, manual palpation, and micro-computed tomography (CT) analysis after excision at 6 weeks. Histological sections of randomly selected spines were subjected to semiquantitative histopathological scoring for bone formation.

Results: By manual palpation, posterolateral fusion was detected in 40\% (6/15) of spines implanted with syngeneic bone, whereas spines implanted with Trinity Evolution and Trinity ELITE allografts yielded 71\% (10/14) and $77 \%$ (10/13) fusion, respectively. Only 7\% (1/14) of spines implanted with Osteocel Plus allografts were judged fused by manual palpation (statistically significantly less than ELITE, $P<.0007$, and Evolution, $P<.0013$ ). The mineralized cancellous bone component of the allografts confounded radiographic analysis, but Trinity Evolution $(0.452 \pm 0.064)$ and Trinity ELITE $(0.536 \pm 0.109)$ allografts produced statistically significantly higher bone fusion mass volumes measured by quantitative micro-CT than did syngeneic bone $(0.292 \pm 0.109, P<.0001$ for ELITE and $P<.003$ for Evolution) and Osteocel Plus $(0.258 \pm 0.103, P<.0001)$. Semiquantitative histopathological scores supported these findings because the total bone and bone marrow scores reflected significantly better new bone and marrow formation in the Trinity groups than in the Osteocel Plus group.

Conclusions: The Trinity Evolution and Trinity ELITE cellular bone allografts were more effective at creating posterolateral fusion than either the Osteocel Plus allografts or syngeneic bone in this animal model.

Clinical Relevance: The superior fusion rate of Trinity cellular bone allografts may lead to better clinical outcome of spinal fusion surgeries.

Biologics

Keywords: Posterolateral spine fusion, athymic rat, stem cells, allograft, Trinity Evolution, Trinity ELITE, Osteocel Plus
\end{abstract}

\section{INTRODUCTION}

Spinal fusion is one of the most commonly used procedures for treating spinal conditions including deformity, trauma, degenerative disc disease, and spondylolisthesis. Autograft iliac crest bone is still considered the "gold standard" graft material for spinal fusion but is associated with increased operative time and blood loss, donor site pain, and infection. ${ }^{1,2}$ There is also often an insufficient quantity of autograft, particularly for long-segment surgeries. ${ }^{2}$ Thus, bone substitutes have been developed including demineralized bone matrices, synthetic implants, and recombinant growth factors. ${ }^{3,4}$ Although these bone grafts are all osteoconductive and some have osteoinductive properties, none contain living, bone-forming cells and therefore cannot be considered to be osteogenic.

In recent years, other substitutes such as allogeneic cell bone grafts have been commercialized. ${ }^{5}$ These are prepared from donated human bone recovered by tissue banks, processed with the purpose of preserving live osteogenic cells in an allograft that retains its natural osteoconductive and osteoinductive properties. Due to the proprietary nature of the processes used to create commercial products, the exact cell and carrier procurement and preparations may vary, affecting the biological efficacy of these products. In this study, we tested 3 commercially available allogeneic cell or matrix 
preparations to determine whether there is significant variability among products. The versions tested were Trinity Evolution ${ }^{\circledR}$ and Trinity ELITE ${ }^{\circledR}$ allografts from Orthofix Medical Inc (Lewisville, TX) and Osteocel ${ }^{\circledR}$ Plus allografts from NuVasive, Inc (San Diego, CA). The study compared the ability of these allografts to make bone and form effective fusions in the athymic rat model of posterolateral spinal fusion.

\section{MATERIALS AND METHODS}

\section{Experimental Groups}

All procedures were done under an Institutional Animal Care and Use Committee-approved protocol. Three-week old male athymic $(\mathrm{rnu} / \mathrm{rnu})$ rats were purchased from the in-house colony of Foxn $1^{r n u}$ rats, in which heterozygous females (rnu/ + ) are bred to homozygous males (rnu/rnu) in stable breeding pairs. The rats aged a further 5 to 6 weeks. Fifteen rats were used for each of the 4 implants: Trinity Evolution, Trinity ELITE, Osteocel Plus allografts, and syngeneic bone. For the last group, iliac crest bone from 15 donor athymic rats was harvested, 1 donor per experimental animal. The operated animals were randomly assigned to each implant type, and there were no differences among the mean weights of the 4 groups of rats at the time of surgery. Because it is possible that differences in donors could result in differences in the implants' osteogenicity, we acquired implants originating from 3 different donors for each commercial allograft and allografts from each donor were implanted in 5 rats to allow an intra-allograft comparison. There were no donor-to-donor differences for manual palpation, $\mathrm{x}$-ray, or micro-computed tomography (CT) radiography for any of the implants.

\section{Surgical Procedure}

Athymic rats were anesthetized with buprenorphine $(0.05-0.01 \mathrm{mg} / \mathrm{kg})$ and maintained on isofluorane during operations. After betadine and alcohol preparation of a rat's dorsal skin, a single midline longitudinal incision of 2 to $3 \mathrm{~cm}$ was made. A separate longitudinal fascial incision was then made over the intertransverse processes on both sides of the L4 and L5 vertebrae. Using a longitudinal muscle-splitting approach, the transverse processes of L4 and L5 and intertransverse membranes were exposed and the former scraped with a periosteal elevator. The cellular allografts were thawed and rehydrated as per each manufacturer's instructions. All implantations occurred within the manufacturers' time allowance for use after preparation. Each implant was placed directly over and between the 2 transverse processes, bilaterally, 1 treatment per rat, using $0.3-\mathrm{mL}$ volume per implant per side. The fascial incisions were closed with 4-0 absorbable suture and the skin stapled. Postsurgery, the rats were allowed free cage movement and food and drink ad libitum.

\section{Necropsy}

Animals were humanely euthanized 6 weeks postoperatively by carbon dioxide asphyxiation. Once death was established by the absence of corneal reflexes and heart tomes (cardiac arrest), the experimental site was recovered. The lumbar segment was harvested by resecting through the L1L2 and a midsacral intervertebral disc space and facet joints.

\section{X-ray Analysis}

Fusion was judged by the presence of continuous bone between the adjacent transverse process by anteroposterior cabinet x-rays (Faxitron, Tucson, AZ) as described previously ${ }^{6-9}$; a scale of 0 to 4 was used, with 0 representing no bone present between the transverse processes and 4 representing bilateral fusion. A spine was determined to be fused only if bone was continuous between the processes bilaterally.

\section{Manual Palpation}

Manual palpation was performed to demonstrate presence of motion at the surgical site. An independent examiner blinded to the treatment type assessed all the spines in a single examination period for fusion in this manner. Presence of motion at the fusion site was scored as 0 , and a stiff spine without obvious motion was recorded as 1 .

\section{Micro-CT}

High-magnification, 59- $\mu \mathrm{m}$ voxel size, 2-minute scans of each spine were conducted in a Quantum FX micro-CT imaging system (PerkinElmer, Waltham MA). Three-dimensional reconstruction images of cut-plane views were created with an image reconstruction software program (OsiriX, V.4.1.2, Pixmeo Sarl, Bernex, Switerland). The bone mass from the upper margin of L4 to the lower margin of 


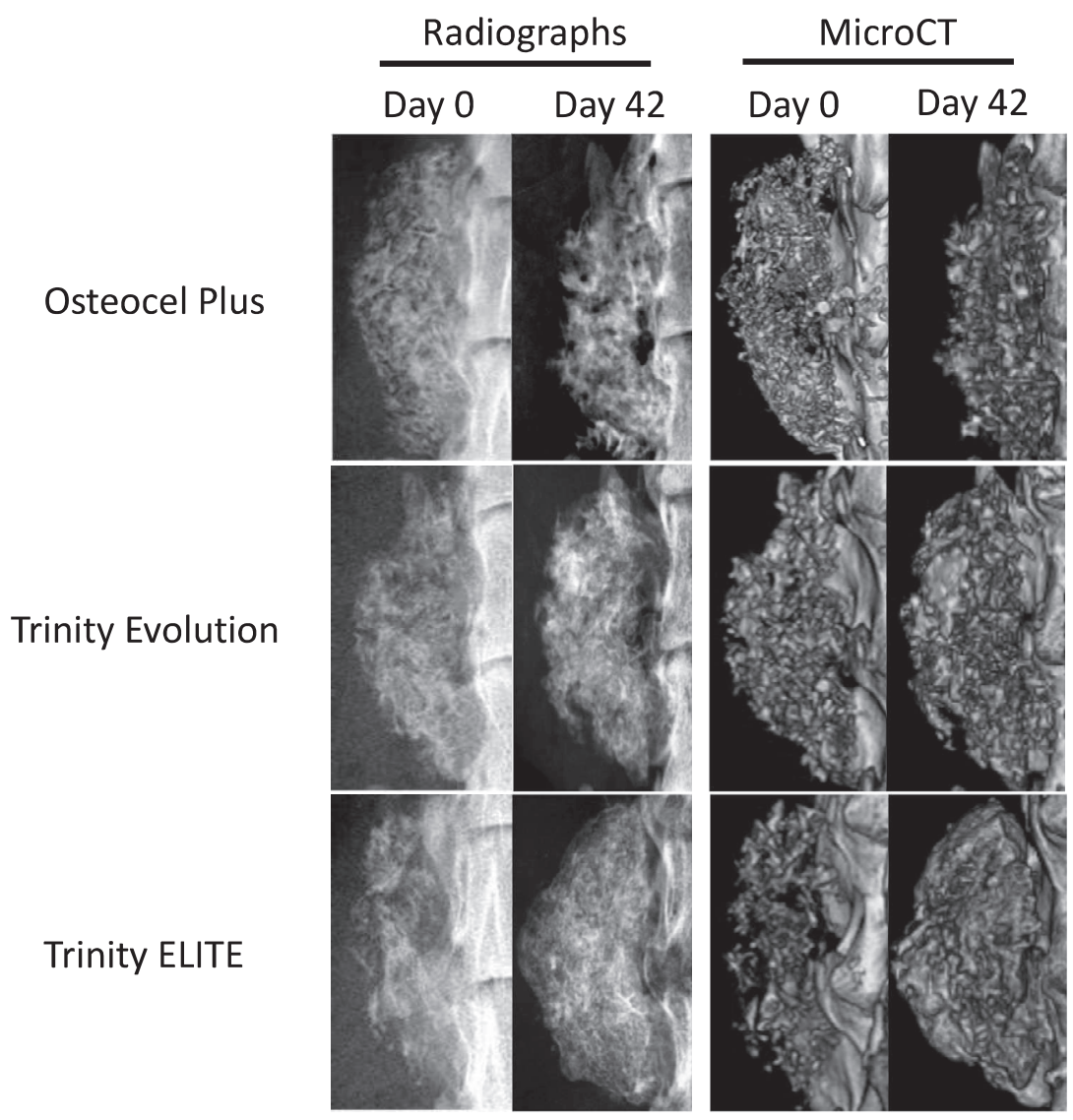

Figure 1. Radiographs and micro-CT 3-dimensional reconstructions of Osteocel Plus, Trinity Evolution, and Trinity ELITE implants immediately after implantation (day 0) and at 6 weeks postimplantation (day 42).

L5 was measured with the same software. The actual fusion mass bone volume was calculated by subtracting the mean bone volume of the L4 and L5 spinous processes and vertebral bodies measured in 5 unoperated spines of male rats of the same weight range as the operated rats.

\section{Histology}

To qualitatively evaluate signs of infection, adverse tissue reaction, and bone and cartilage formation, 2 randomly selected specimens from each group were fixed in $10 \%$ formalin. These were decalcified with EDTA, embedded in paraffin, and sectioned and stained with toluidine blue. For each spine, 10 to 15 slides were cut and stained per side. Representative images were taken and are presented in Figure 3.

A semiquantitative histological scoring scheme (Table 1) was used to assess the bone formation at 6 weeks. ${ }^{10}$ The evaluation was performed at Histion LLC (Everett, WA) by an evaluator completely blinded to the experimental groups during the assessment. Three randomly selected spines were fixed in $10 \%$ neutral buffered formalin, halved down the midline in the sagittal plane, and embedded in paraffin. Two sections were then taken from each side of the spines, stained with toluidine blue, evaluated, and scored microscopically by the experienced reviewer blinded to the study. Scores from the 2 sections of each side of the spine were averaged and treated as 1 independent observation during the statistical analysis. The scores of the woven bone and lamellar bone from each section were summed together as a total bone score.

\section{STATISTICAL METHODS}

The contingency table and Fisher exact method were used to compare differences in proportions among groups for the manual palpation of fusion. For the bone volume micro-CT analysis, analysis of variance was used for group comparisons. Furthermore, the Student $t$ test was performed for the semiquantitative histopathological comparisons. Significance for analysis of variance and Student $t$ test were set at $P<.05$. 

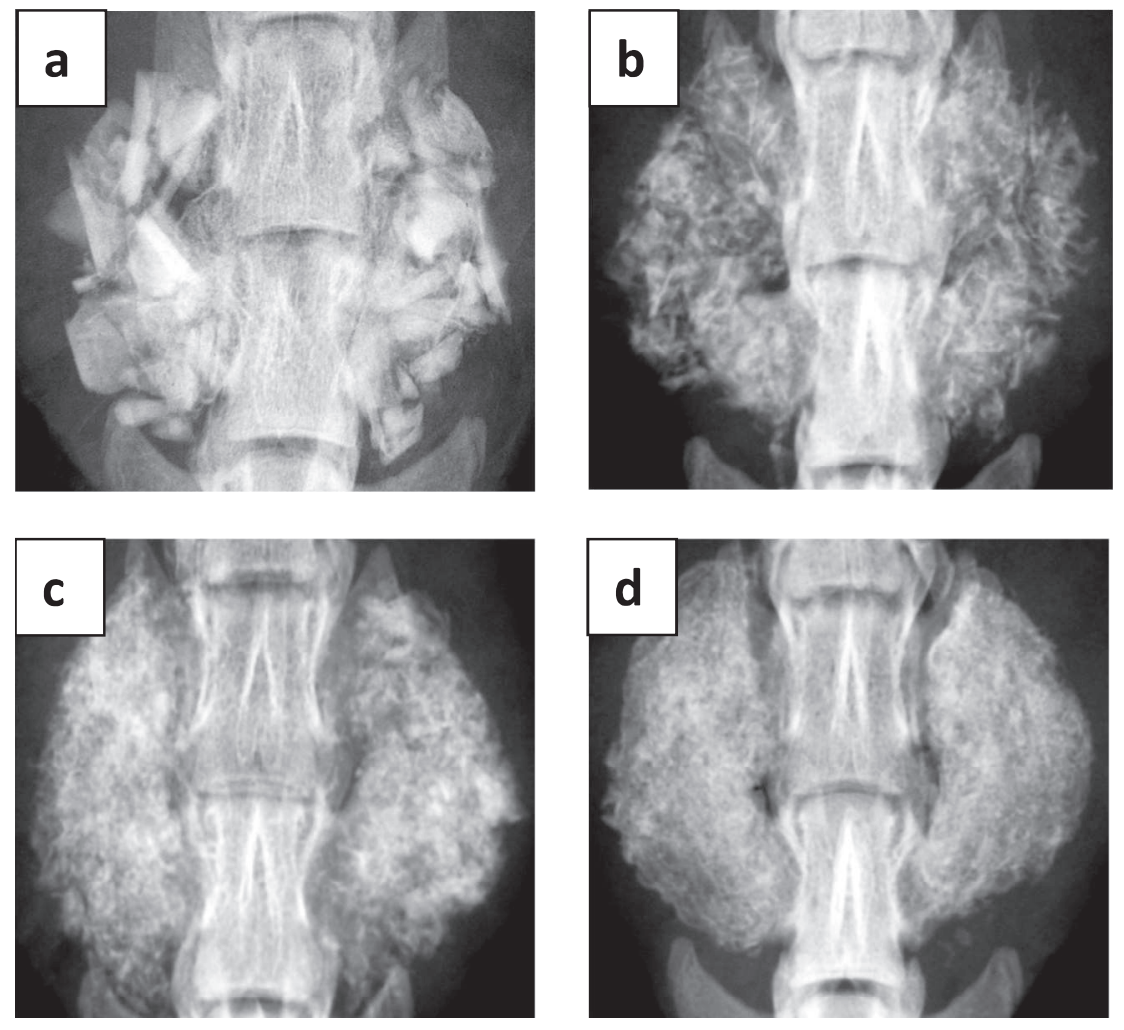

Figure 2. Radiographs of (a) syngeneic bone, (b) Osteocel Plus, (c) Trinity Evolution, and (d) Trinity ELITE allografts 6 weeks postimplantation in the athymic rat lumbar spine.

\section{RESULTS}

One rat of the syngeneic bone implant group died under anesthesia and was replaced. Four other rats died before the 6-week time point and were not used in the analyses. There were 2 premature deaths in the Trinity ELITE group and 1 each from the Trinity Evolution and Osteocel Plus groups. None

Table 1. Semiquantitative histological scoring scheme for bone formation.

\begin{tabular}{lc}
\hline Formation and Percentage of Space Occupied & Score \\
\hline Woven bone within the implant area & \\
$76-100$ & 4 \\
$51-75$ & 3 \\
$26-50$ & 2 \\
$1-25$ & 1 \\
None & 0 \\
Lamellar bone within the implant area ${ }^{\mathrm{a}}$ & \\
$76-100$ & 4 \\
$51-75$ & 3 \\
$26-50$ & 2 \\
$1-25$ & 1 \\
None & 0 \\
Total bone and bone marrow of the implant area & \\
$76-100$ & 4 \\
$51-75$ & 3 \\
$26-50$ & 2 \\
$1-25$ & 1 \\
None & 0 \\
\hline
\end{tabular}

${ }^{\mathrm{a}}$ Residual implant pieces were included in the assessment.

${ }^{\mathrm{b}}$ Residual implant pieces were excluded from the assessment. of the 4 rats had shown any obvious signs of distress, and the wound sites were without detectable infection both grossly and histologically. One animal from the ELITE group and 1 from the Evolution group underwent a necropsy and a kidney infection was noted. We have seen this in 2 other athymic rats used for separate, unrelated studies (unpublished data). Thus, none of the deaths appeared to be related to the implants used.

Upon manual palpation testing by a single observer blinded as to the implant type used, 6 of $15(40 \%)$ of the syngeneic bone-implanted rats were deemed fused at 6 weeks (Table 2). In comparison, only 1 of $14(7 \%)$ of the Osteocel Plus group was considered fused. These groups were not significantly different from each other. By contrast, 10 of 14 (71\%) Evolution and 10 of 13 (77\%) ELITE spines were judged fused. Whereas neither result was

Table 2. Manual palpation scores for all implants.

\begin{tabular}{lc}
\hline Graft Type & No./Total (\%) Fused \\
\hline Syngeneic bone & $6 / 15(40)$ \\
Osteocel Plus & $1 / 14(7)$ \\
Trinity Evolution & $10 / 14(71)^{*}$ \\
Trinity ELITE & $10 / 13(77)^{* *}$ \\
\hline
\end{tabular}

$* P<.0013 ; * * P<.0007$ compared with Osteocel Plus. 

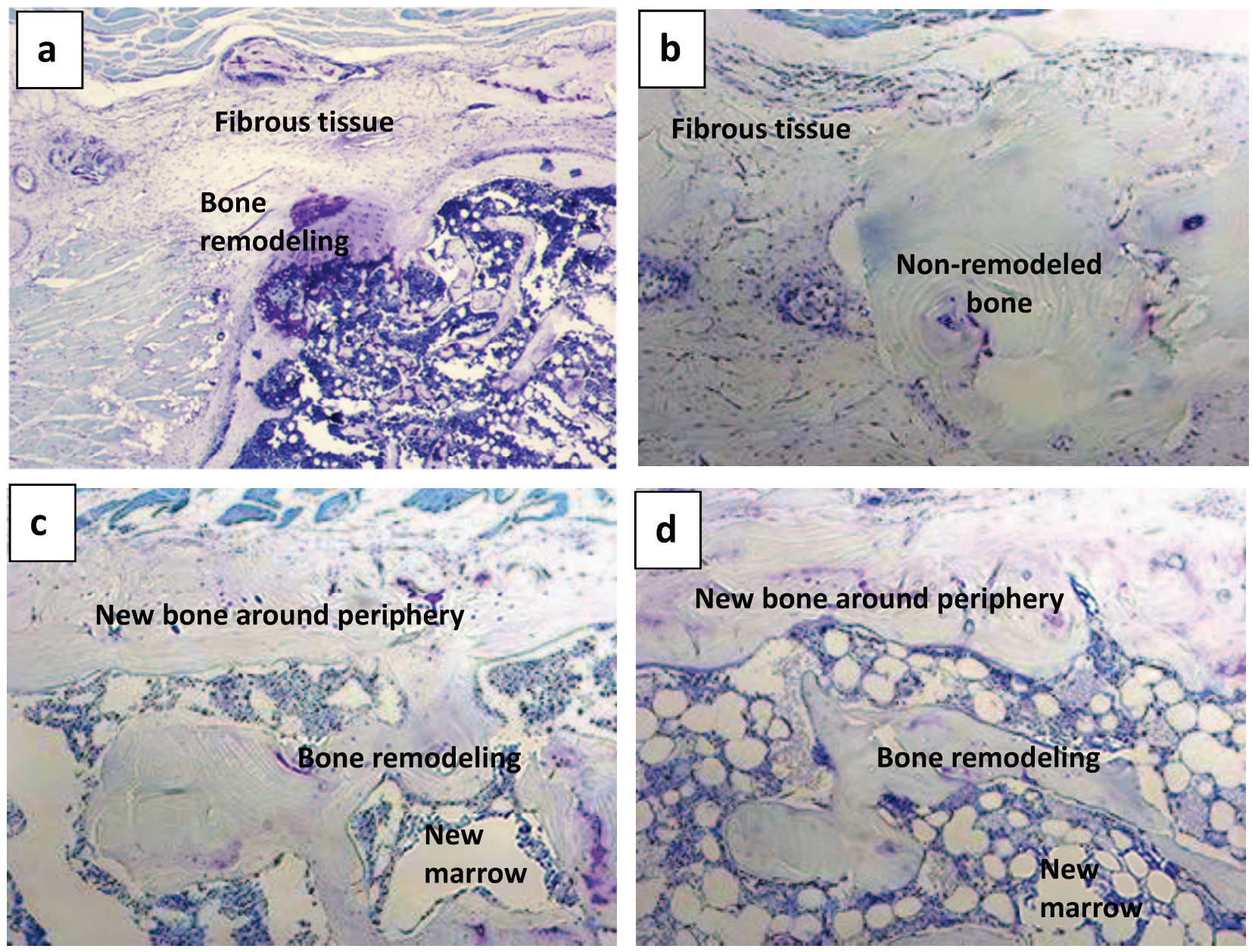

Figure 3. Histology of (a) syngeneic bone (b) Osteocel Plus, (c) Trinity Evolution, and (d) Trinity ELITE allografts 6 weeks postimplantation in the athymic rat lumbar spine (toluidine blue-stained, paraffin-embedded sections).

statistically different from the $40 \%$ rate for syngeneic bone, both were significantly higher than the Osteocel Plus group $(P<.0013$ and $P<.0007$ for Evolution and ELITE, respectively).

Mineralized bone graft material remained from the original implants in all of the animals when radiographs were analyzed, and bilateral fusion (a score of 4) was observed for all groups. Example radiographs in Figures 1 and 2 indicate that there was some remodeling of the implants, with clearly defined fusion masses with smoother edges seen in the Evolution and ELITE groups. Because this is a qualitative assessment, it cannot easily be used for comparisons of the groups. However, the histological results correlated with this observation (Figure 3): the fusion masses of the Evolution and ELITE groups had more defined bony borders and remodeled interiors containing greater new marrow formation, whereas the Osteocel Plus fusion masses had mostly fibrous tissue surrounding unremodeled bone with less evidence of new bone and marrow formation. The analysis of the semiquantitative histopathological scoring indicated that there were no statistical differences among the 4 types of implants in terms of the total bone scores (sum of woven and lamellar bone) within the implant area when the residual graft materials were included into the assessment (Figure 4). However, with the residual graft materials excluded, the total bone and bone marrow scores for the syngeneic bone, Trinity Evolution, and ELITE allografts were all statistically significantly higher than that of Osteocel Plus allografts.

Furthermore, quantitative data that correlated with these findings were attained from the micro-CT analysis (Figure 5). The Trinity Evolution and ELITE fusion masses had significantly higher bone volumes than those of syngeneic bone $(P<.003$ for Evolution and $P<.0001$ for ELITE) and the Osteocel Plus groups $(P<.0001$ for both groups $)$ at 


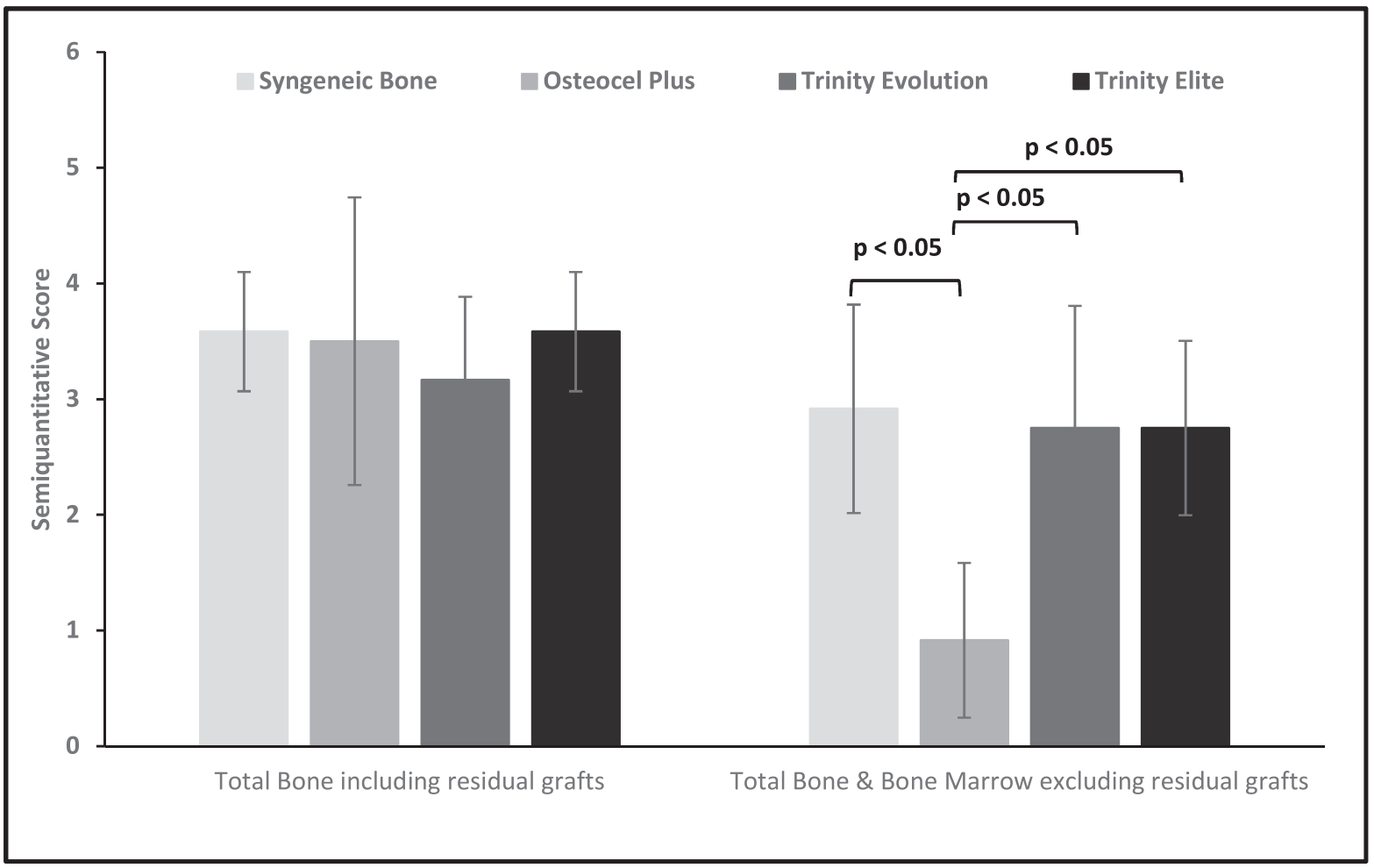

Figure 4. Semiquantitative histopathological scores for syngeneic bone, Osteocel Plus, Trinity Evolution, and Trinity ELITE allografts 6 weeks postimplantation in the athymic rat lumbar spine.

6 weeks. There was no significant difference between the volumes of the 2 Trinity graft types.

\section{DISCUSSION}

In this study, we sought to provide the first direct in vivo comparison of viable cell-containing bone allografts on the market. In previous studies, we have produced rabbit versions of nonviable human allografts to enable testing in a bone paucity model of posterolateral spine fusion we developed. ${ }^{7}$ This is more challenging with allograft formulations that contain living cells because species variation in stem and osteogenic cell populations and frequency are difficult to account for. Therefore, we used the athymic rat model of posterolateral spine fusion that has been used for studies with xenogeneic implants. ${ }^{11}$ There is no difference between athymic and normothymic rat fusion rates. ${ }^{12}$ However, this model does have the drawback that autologous bone does not provide as high a fusion rate as that described clinically. Grauer et $\mathrm{al}^{12}$ achieved only a $30 \%$ manual palpation fusion rate after 6 weeks and $10 \%$ radiographically with 0.1 to $0.2 \mathrm{~mL}$ autologous iliac crest bone, much lower than the $60 \%$ to $70 \%$ rate in humans. Wang et al did not achieve any fusion by manual palpation or radiography using
$0.3 \mathrm{~mL}$ of either autologous rat tail bone ${ }^{13}$. Similar results were observed with iliac crest bone by Lee et al. ${ }^{14}$ The physical space between and above rat vertebrae limits the amount of bone that can be implanted. This notwithstanding, even taking 0.3 $\mathrm{mL}$ of crest bone is a considerable harvest that has the potential for morbidity. Thus, we substituted syngeneic iliac crest bone from littermates, implanting $0.3 \mathrm{~mL}$ per side to match the volume of the cellular allografts and achieved a 40\% manual palpation fusion rate.

Another limitation of the study is the minimal utility of radiographic fusion scoring when radiodense material is implanted. ${ }^{12-14}$ Overestimating radiographic fusion is a noted problem regardless of the starting material. ${ }^{8,14}$ Although sensitivity and negative predictive value can be high, positive predictive value is poor even for fine-detail radiographs. ${ }^{8}$ From the histological findings in this study, residual graft pieces left at the implantation site after 6 weeks of bone remodeling appeared different from the newly formed woven and lamellar bone in terms of their stained color, morphology, and location. Therefore, the newly formed bone and bone marrow could be semiquantitatively scored, excluding the residual graft pieces. This score for the 


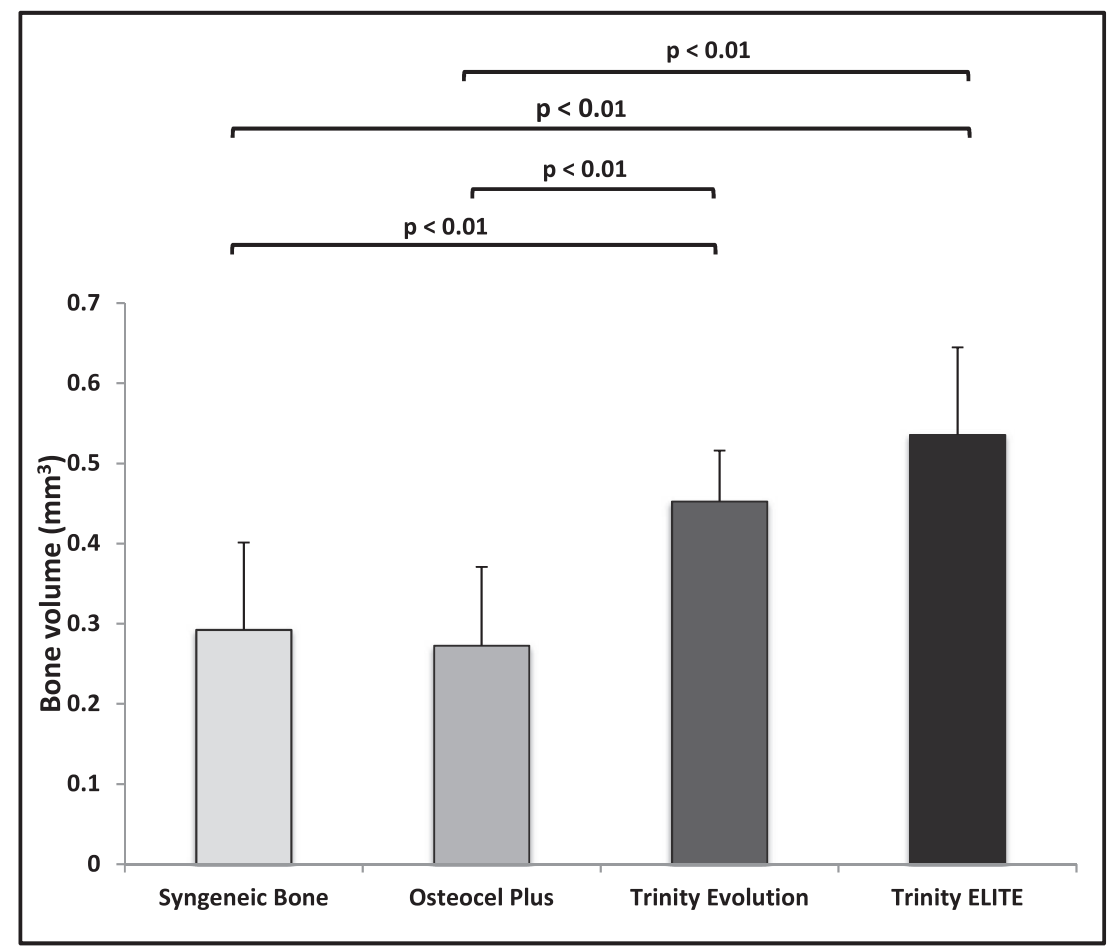

Figure 5. Volume of fusion masses $\left(\mathrm{mm}^{3}\right)$ for syngeneic bone, Osteocel Plus, Trinity Evolution, and Trinity ELITE allografts 6 weeks postimplantation in the athymic rat lumbar spine determined from micro-CT images.

total bone and bone marrow is conceptually a better approach to evaluate the ability of promoting new bone formation by each allograft, compared with a total bone score where the residual graft pieces were included in the assessment. A significantly lower total bone and bone marrow score for Osteocel Plus allografts indicated that its ability to enhance new bone formation and bone remodeling is not as effective as the 2 Trinity allografts. Indeed, histopathological observation under microscope confirmed that Osteocel Plus allografts contained mainly nonremodeled graft bone, whereas formation of a discrete fusion mass with more evidence of marrow formation was seen in the Trinity Evolution and ELITE groups. These results correlated with the more defined fusion masses observed radiographically for the Trinity implants.

Micro-CT images were used to quantify the volume of bone in the fusion masses, which includes both the newly formed bone and residual graft implants. Implantation of either Trinity Evolution or ELITE allografts resulted in bone volumes 6 weeks later that were statistically significantly greater than those that resulted from implantation of either Osteocel Plus allografts or syngeneic bone, which did not differ from each other. This increased bone in the Trinity fusion masses was not confirmed by the total bone scores of the histopathological assessment; the discrepancy is possibly due to the limitations of this semiquantitative scoring scheme. It suffers from a lack of sensitivity because there is a $25 \%$ scoring spread for the same scoring integer. The same problem likely occurs in the total bone and bone marrow assessment, where different scores could be assigned to similar percentage numbers likely falling just across the line that differentiates 2 scores. In this case, the resulting maximum score difference is 1 . However, the actual differences in total bone and bone marrow scores between Osteocel Plus implants and the 3 other implant types are more than 1.8, indicating the observable differences in the new bone and bone marrow coverage within the implant area are appropriately captured.

There is a paucity of literature on the efficacy of viable cell bone allografts for tissue repair. The Trinity Evolution and Osteocel allografts have been evaluated separately in 3 clinical studies for foot and ankle arthrodesis and lumbar interbody fusion. ${ }^{15-17}$ However, neither side-by-side comparisons of these products nor comparisons with autograft controls have been performed. Our study indicates that allograft cell preparations do not all perform the same in vivo. Due to the proprietary nature of the 
preparations of these products, it is difficult to theorize as to the reasons for this variability. It is possible that even small differences in processing could affect the native progenitor population, chemical compositions of the extracellular matrix, and physical properties of these allografts. Because large animal models that are immune-compromised are not available, we are limited in the preclinical assessments that can be carried out with cell-based allografts. Whereas this study indicates that at least some of the first marketed cellular bone matrix allografts can be effective at generating bony fusions in the posterolateral spine in vivo, caution should be taken when translating the current animal results into clinical outcome of human subjects. A more comprehensive evaluation would require well-controlled prospective clinical studies for a complete understanding of their efficacy in relation to the human gold standard autologous graft and other allograft alternatives. However, such studies are expensive and difficult to execute. The present study represents one of the few alternatives we have to a clinical trial, given that it at least allows the use of the actual commercial products rather than surrogate or similarly prepared versions that would have to be used in larger animal models.

Allogeneic bone matrices containing live osteogenic cells represent the most recent allograft technology that has been developed as an alternative to autologous iliac crest bone. In this study, we have found that Trinity Evolution and Trinity ELITE cellular allografts produce significantly better posterolateral spinal fusion at 6 weeks in an athymic rat model than implanting the equivalent amount of either syngeneic bone or Osteocel Plus cellular allografts. It is not known yet whether these differences still hold true beyond the 6-week time point and how they can be interpolated to the clinical outcome of surgery using these types of implants, but the study indicates that different cellular bone allografts are not equivalent in their capacity to effect spinal fusion.

\section{REFERENCES}

1. Kurz LT, Garfin SR, Booth RE Jr. Harvesting autogenous iliac bone grafts. A review of complications and techniques. Spine (Phila Pa 1976). 1989;14(12):1324-1331.

2. Park JJ, Hershman SH, Kim YH. Updates in the use of bone grafts in the lumbar spine. Bull Hosp Jt Dis (2013). 2013;71(1):39-48.

3. Vaccaro AR, Chiba K, Heller JG, et al. Bone grafting alternatives in spinal surgery. Spine J. 2002;2(3):206-215.
4. Rihn JA, Kirkpatrick K, Albert TJ. Graft options in posterolateral and posterior interbody lumbar fusion. Spine (Phila Pa 1976). 2010;35(17):1629-1639.

5. Skovrlj B, Guzman JZ, Al M, M., Cho SK, Latridis JC, Qureshi SA. Cellular bone matrices: viable stem cell-containing bone graft substitutes. Spine J. 2014;14(11):2763-2772.

6. Choi Y, Oldenburg FP, Sage L, Johnstone B, Yoo JU. A bridging demineralized bone implant facilitates posterolateral lumbar fusion in New Zealand white rabbits. Spine (Phila Pa 1976). 2007;32(1):36-41.

7. Curylo LI, Johnstone B, Petersilge CA, Janicki JA, Yoo JU. Augmentation of spinal arthrodesis with autologous bone marrow in a rabbit posterolateral spine fusion model. Spine. 1999;24(5):434-438, discussion 438-439.

8. Yee AJ, Bae HW, Friess D, Robbin M, Johnstone B, Yoo JU. Accuracy and interobserver agreement for determinations of rabbit posterolateral spinal fusion. Spine (Phila Pa 1976). 2004;29(12):1308-1313.

9. Yee AJ, Bae HW, Friess D, et al. The use of simvastatin in rabbit posterolateral lumbar intertransverse process spine fusion. Spine J. 2006;6(4):391-396.

10. Corre P, Merceron C, Vignes C, et al. Determining a clinically relevant strategy for bone tissue engineering: an "allin-one" study in nude mice. PloS One. 2013;8(12):e81599.

11. Boden SD, Titus L, Hair G, et al. Lumbar spine fusion by local gene therapy with a cDNA encoding a novel osteoinductive protein (LMP-1). Spine (Phila Pa 1976). 1998;23(23):2486-2492.

12. Grauer JN, Bomback DA, Lugo R, Troiano NW, Patel TC, Friedlaender GE. Posterolateral lumbar fusions in athymic rats: characterization of a model. Spine J. 2004;4(3):281-286.

13. Lee YP, Jo M, Luna M, Chien B, Lieberman JR, Wang JC. The efficacy of different commercially available demineralized bone matrix substances in an athymic rat model. $J$ Spinal Disord Tech. 2005;18(5):439-444.

14. Wang JC, Alanay A, Mark D, et al. A comparison of commercially available demineralized bone matrix for spinal fusion. Eur Spine J. 2007;16(8):1233-1240.

15. Ammerman JM, Libricz J, Ammerman MD. The role of Osteocel Plus as a fusion substrate in minimally invasive instrumented transforaminal lumbar interbody fusion. Clin Neurol Neurosurg. 2013;115(7):991-994.

16. Tohmeh AG, Watson B, Tohmeh M, Zielinski XJ. Allograft cellular bone matrix in extreme lateral interbody fusion: preliminary radiographic and clinical outcomes. Scientific WorldJournal. 2012;2012:263637.

17. Jones CP, Loveland J, Atkinson BL, Ryaby JT, Linovitz RJ, Nunley JA. Prospective, multicenter evaluation of allogeneic bone matrix containing viable osteogenic cells in foot and or ankle arthrodesis. Foot Ankle Int. 2015;36(10):1129-1137.

Disclosures and COI: This study was funded by a research grant received by B.J. and J.Y. from Orthofix Medical Inc (Lewisville, TX) and MTF Biologics (Edison, NJ). Because MTF Biologics and Orthofix Medical Inc are the manufacturer and distributor of the Trinity spine allografts, we mitigated the potential conflict of interest by blinding every aspect of the study and carrying out 
the procedures and analyses in this manner before breaking the codes used and examining any differences between the results for the various implants. This process included surgeons not being aware of which allograft they were implanting; randomization of implantation order between each 5-rat batch used for a given implant; manual palpation scoring, x-ray scoring, and micro-CT volume measurements done with all spines randomized and blinding of scorers as to implant type; histology descriptions made by researchers blinded as to implant type. J.T.R., E.I.W., and N.Z. are employees of and own stocks in Orthofix Medical Inc. E.S. and A.D. are employees of MTF Biologics.

Corresponding Authors: (1) Brian John- stone, PhD, Department of Orthopaedics and Rehabilitation, Oregon Health \& Science University, 3181 SW Sam Jackson Park Rd, Portland, OR 97202. Phone: (502) 494-9505; Fax: (503) 494-5050; Email: johnstob@ohsu.edu. (2) Nianli Zhang, PhD, Research \& Clinical Affairs, Orthofix Medical Inc, 3451 Plano Parkway, Lewisville TX 75056. Phone: (214) 937-2377; Email: nianlizhang@orthofix.com.

Published 30 April 2020

This manuscript is generously published free of charge by ISASS, the International Society for the Advancement of Spine Surgery. Copyright (C) 2020 ISASS. To see more or order reprints or permissions, see http://ijssurgery.com. 